\title{
Eccema numular: reporte de tres casos
}

Nummular eczema: Report of three cases

\section{María Alejandra Casas-Barrera' ${ }^{1}$, José William Ricardo-Soto ${ }^{2}$, Mariam Carolina Rolón- Cadena ${ }^{3}$, Juan Guillermo Chalela-Mantilla ${ }^{4}$}

1. Médica general, Hospital Universitario Fundación Santa Fe de Bogotá, Bogotá, Colombia

2. Médico interno institucional, Hospital Universitario Fundación Santa Fe de Bogotá, Universidad de los Andes, Bogotá, Colombia

3. Médica dermatopatóloga, Hospital Universitario Fundación Santa Fe de Bogotá, Bogotá, Colombia

4. Médico internista, dermatólogo, Asociación Médica de los Andes, Bogotá, Colombia

\section{RESUMEN}

El eccema numular es una dermatosis crónica y recidivante que se manifiesta con placas en forma de moneda, pruriginosas, de tamaño variable y bordes definidos, que evolucionan a placas liquenificadas y con descamación. A pesar de que se considera una enfermedad de adultos, existen reportes de caso de niños con eccema numular. Afecta a ambos sexos y las lesiones se presentan con mayor frecuencia en las extremidades, aunque pueden comprometer el tronco y el rostro.

La etiología de esta entidad es desconocida. Se considera una enfermedad multifactorial en la que intervienen fármacos, infecciones, alérgenos, irritantes, factores ambientales y factores emocionales, entre otros. Dado que la histopatología es inespecífica, el diagnóstico es eminentemente clínico.

Los tratamientos farmacológicos más usados y reportados involucran corticoides tópicos de mediana y gran potencia, asociados a emolientes y antihistamínicos. Sin embargo, hay reportes de casos en los que se han usado inmunomoduladores e inmunosupresores, especialmente en los casos graves y de difícil manejo.

PALABRAS CLAVE: eccema, dermatitis atópica, dermatitis por contacto.

\section{SUMMARY}

Nummular eczema is a chronic and relapsing dermatosis that presents with a pruritic, well-demarcated plaques that are coin-shaped and vary in size; these plaques often evolve into lichenified patches. Even though this is often referred to an adult issue, there have been reported cases in children. Nummular eczema affects both sexes, and lesions often present in the lower extremities; however, it can also affect the torso and face.

Etiology is unknown: there are several triggering agents that affect pathology which include but are not limited to medications, allergens, environmental irritants, and/or emotional stress. Nummular eczema is diagnosed clinically due to its uncertain pathophysiology.

The more commonly used treatment involves topical corticosteroids of medium and long range, along with emollients and antihistamines. Nonetheless, there have been reports in which immunosuppressants and immunomodulators have been used in severe and hard to manage cases.

KEY WORDS: Eczema, atopic dermatitis, contact dermatitis.
Correspondencia:

María Alejandra Casas-Barrera

Email:

m_casas94@hotmail.com

Recibido: 08/08/18

Aceptado: 27/02/19

Conflictos de interés:

No se reportan conflictos de interés.

Financiación:

Ninguna. 


\section{CASOS CLÍNICOS}

\section{Caso 1}

Se trata de un hombre de 72 años, con antecedentes de hipotiroidismo, osteoporosis y gastritis crónica, en tratamiento con levotiroxina, carbonato de calcio, aspirina y omeprazol. El paciente consultó por presentar lesiones cutáneas circulares, eritematosas y pruriginosas en la espalda, de dos años de evolución. Refería haberse aplicado desonida en crema al 0,05 \% sobre las lesiones de manera irregular, sin obtener mejoría clínica.

En la exploración física, se encontraron múltiples placas eritematosas, con descamación, con tendencia a la liquenificación, de borde papular y superficie central aplanada, algunas excoriadas, ubicadas con un patrón bilateral y simétrico, principalmente en la espalda (figura 1) y en la cara anterior de los miembros superiores. Además, presentaba una lesión de aparición reciente y de características similares, en el pliegue poplíteo externo derecho.

En la biopsia de las lesiones se reportó una epidermis acantósica, ortoqueratósica y espongiótica moderada. En la dermis había escaso infiltrado linfocitario con algunos eosinófilos de distribución perivascular su- perficial. La coloración con ácido peryódico de Schiff (PAS) resultó negativa para hongos y descartó la tiña corporal (tinea corporis) como diagnóstico diferencial. Ante la sospecha de eccema numular subagudo, se decidió iniciar tratamiento con prednisona oral, en una dosis de $50 \mathrm{mg} / \mathrm{d}$, durante 1 semana, y se solicitaron exámenes paraclínicos adicionales. A la semana, el paciente evolucionó de manera parcialmente favorable, con mejoría del prurito en un $80 \%$, pero con persistencia de algunas lesiones, principalmente en la espalda, por lo que se continuó el tratamiento con prednisona oral, en una dosis de $25 \mathrm{mg} / \mathrm{d}$, durante 5 semanas.

En los exámenes paraclínicos solicitados, hubo una elevación de la inmunoglobulina E (151,3 UI/ml) y de las proteínas de la región gamma en la electroforesis. Los demás estudios se encontraron dentro de límites normales (glucemia, insulina, creatinina, hemograma, tirotropina [TSH] y perfil lipídico).

Dos semanas después de finalizado el tratamiento, el paciente volvió a consultar por reaparición de lesiones de características similares, ubicadas en la espalda. Se decidió iniciar azatioprina oral, en una dosis de $50 \mathrm{mg}$ tres veces al día, durante 20 días; después, $50 \mathrm{mg}$ dos veces al día, durante 1 mes; y, finalmente, $50 \mathrm{mg} / \mathrm{d}$, durante 1 mes, con lo cual se logró la resolución completa del cuadro clínico hasta el presente.

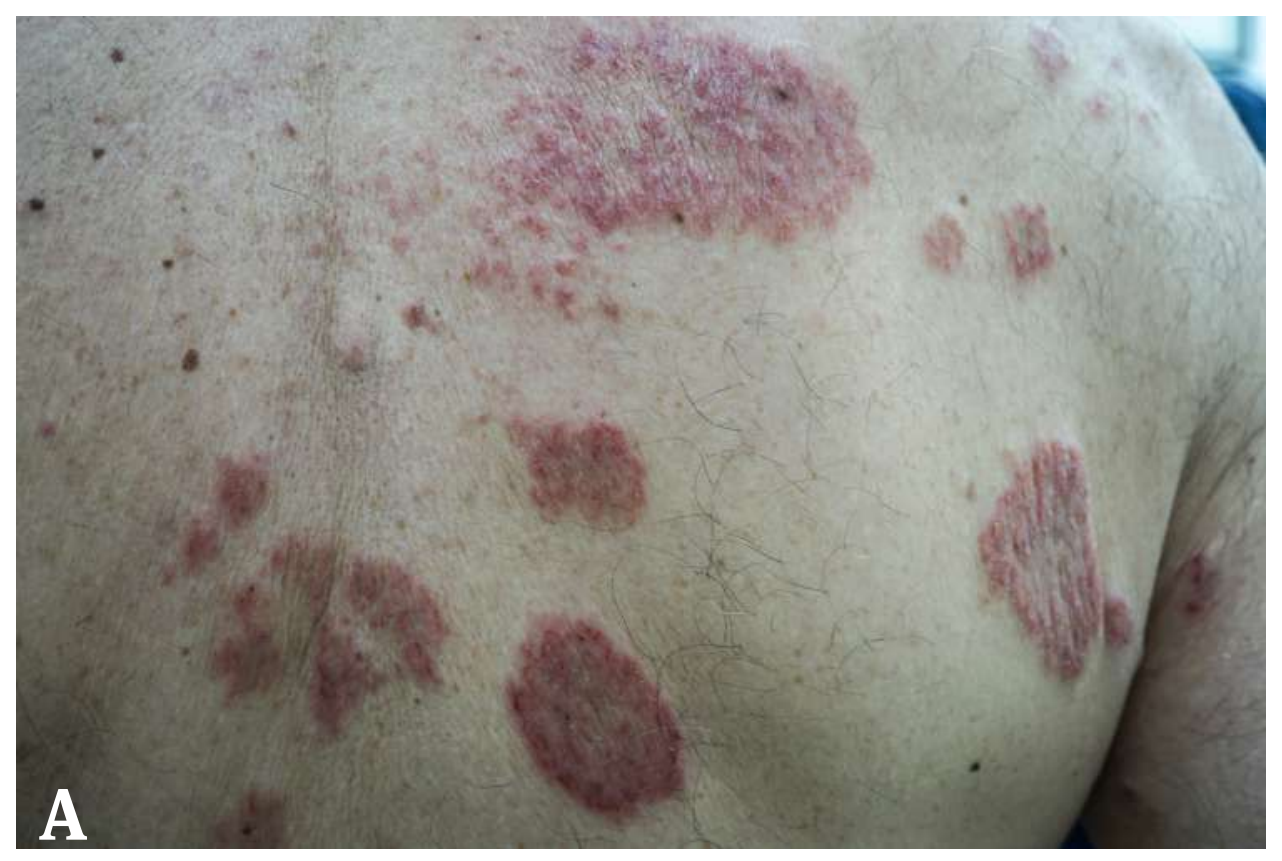

Figura 1. Caso 1. Placas circulares de aspecto eritematoso y descamativo con tendencia a la liquenificación, de borde papular y superficie central aplanada, localizadas en espalda de manera bilateral. A) Vista dorsal. B) Vista dorsal, lado izquierdo. C) Vista dorsal, lado derecho. 

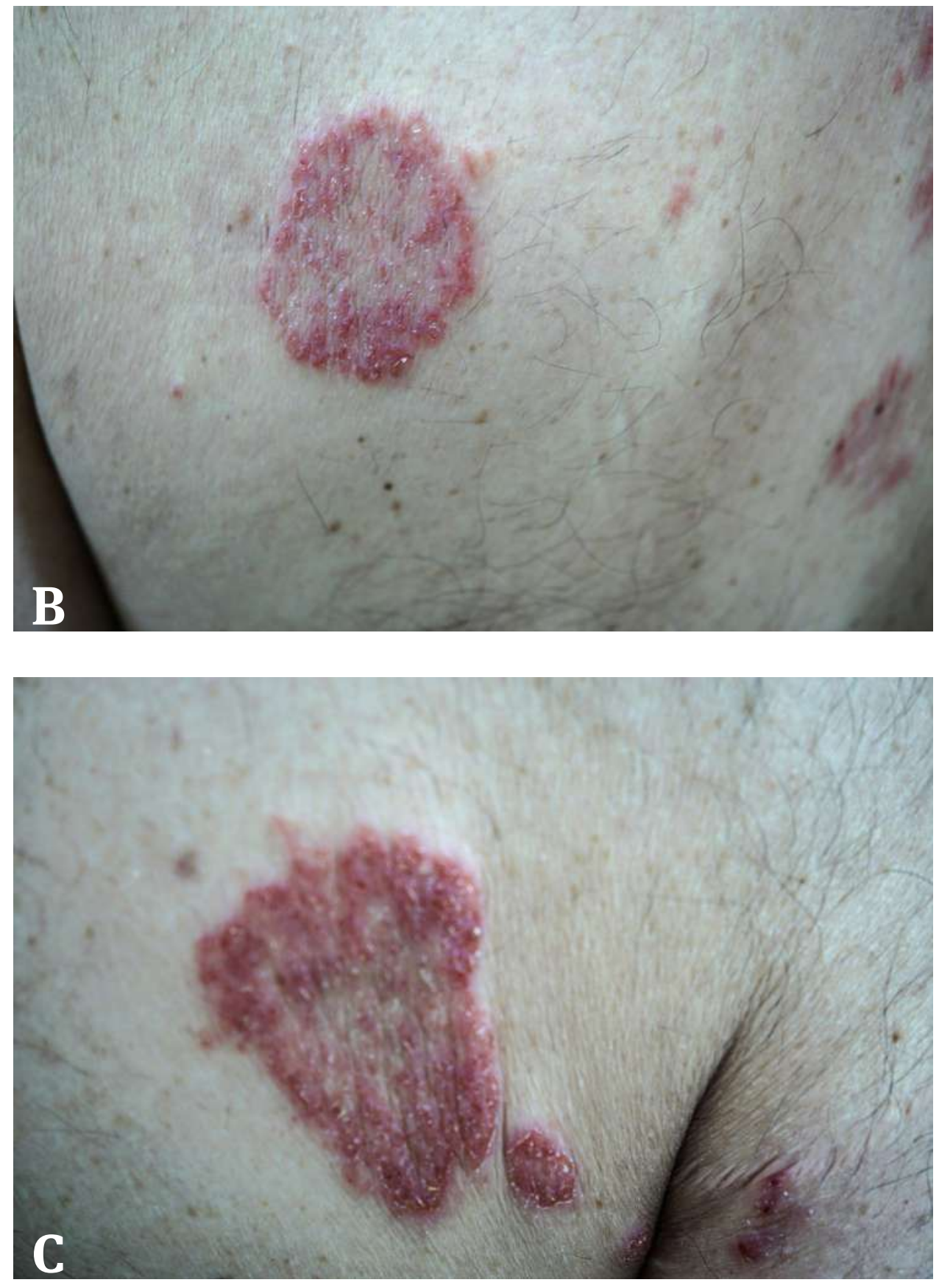


\section{Caso 2}

Se trata de un hombre de 37 años, con antecedentes de asma durante la infancia y pólipos nasales, que consultó por la aparición de unas lesiones eritematosas y pruriginosas en el tronco y las extremidades. Refirió haber consultado previamente a dos dermatólogos diferentes. Inicialmente, fue tratado con rifamicina tópica e hidratante corporal y, posteriormente, loratadina oral, ácido fusídico al 2 \% en crema, moxifloxacino en tabletas y betametasona en crema, ante el diagnóstico de pitiriasis rosada; sin embargo, las lesiones no mejoraron.

En la exploración física, presentaba en ambos miembros inferiores placas eritematosas, circulares, de bordes bien definidos, con descamación, pruriginosas, excoriadas y con costra sérica en su superficie. Tam- bién se observaron múltiples placas de características similares, pero de menor tamaño, ubicadas de forma simétrica en el tronco, los glúteos y los miembros superiores (figura 2).

Se tomó biopsia de las lesiones de la espalda y las piernas, que evidenció una epidermis con espongiosis global importante y una capa córnea delgada con paraqueratosis focal y vesiculillas. En la dermis superficial, se observó infiltrado linfocitario con pocos melanófagos y eosinófilos ocasionales. Además, en la dermis profunda presentaba un infiltrado linfocitario perivascular y perineural.

Dado el cuadro clínico y el resultado de la histopatología del paciente, se consideró un cuadro sugestivo de eccema numular; sin embargo, no se hizo manejo terapéutico ni seguimiento, ya que el paciente no volvió a asistir a la consulta.
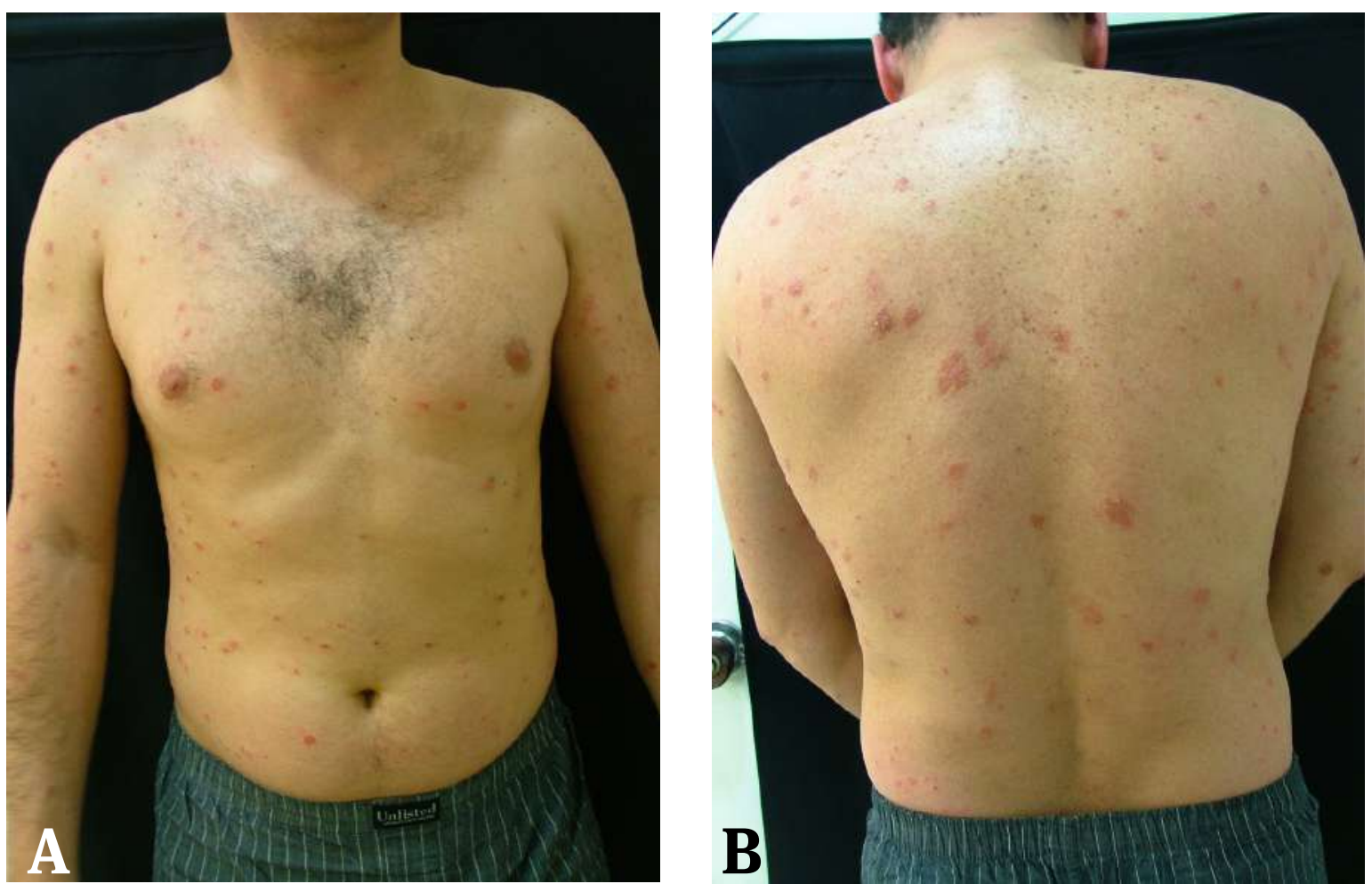

Figura 2. Brote consistente en placas circulares de bordes bien definidos, eritematosas, en fase descamativa, localizadas en el tronco y en las extremidades, de manera simétrica. A) Vista frontal. B) Vista dorsal. C) Piernas. D) Brazo. 

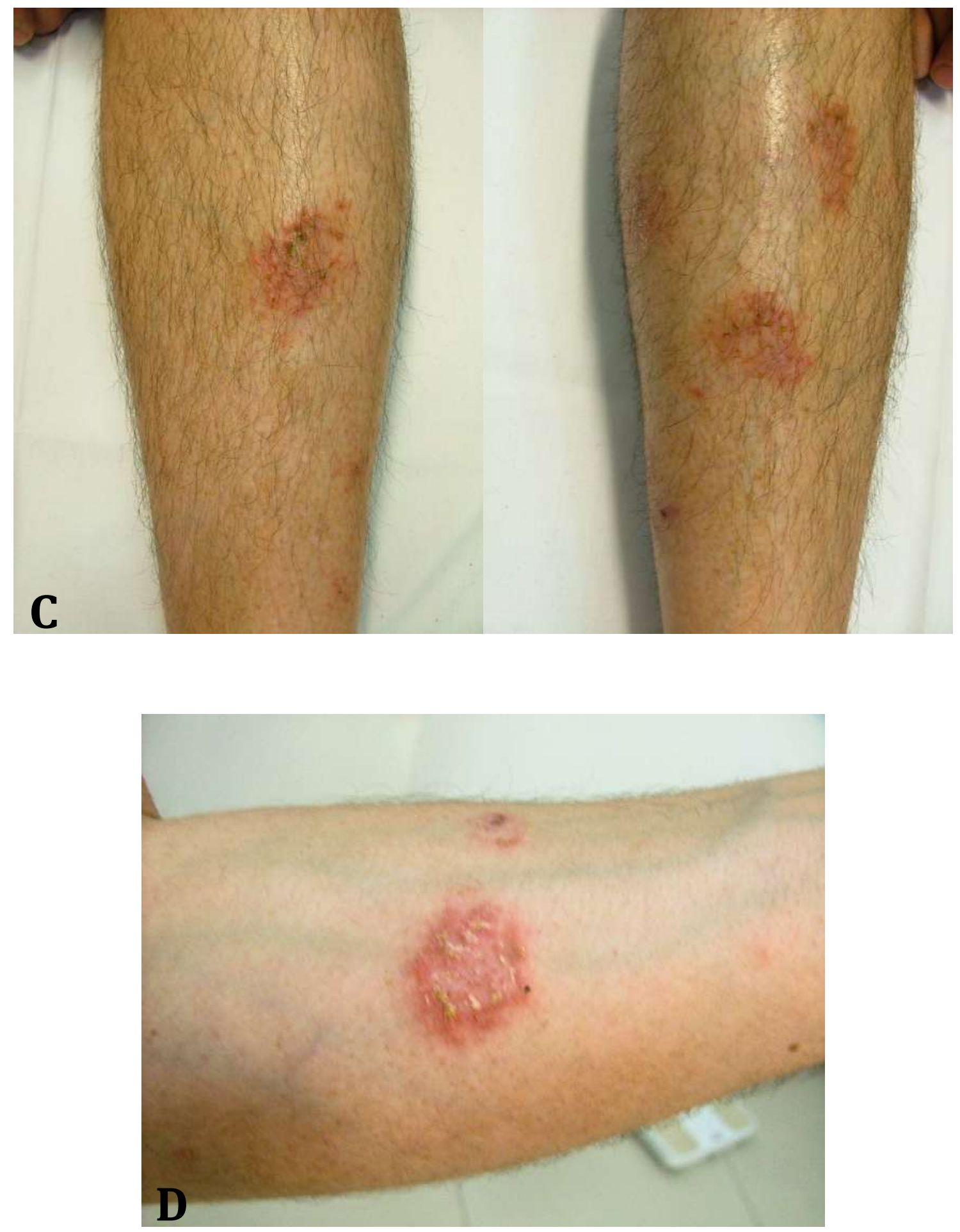


\section{Caso 3}

Se trata de un hombre de 88 años, con antecedentes de cáncer de vejiga tratado, arritmia cardíaca, hipertensión arterial sistémica, enfermedad coronaria y diabetes mellitus. Recibía tratamiento con paroxetina, valsartán, metformina, aspirina y metoprolol. Consultó por prurito generalizado de dos años de evolución, aproximadamente, que lo despertaba durante la noche y alteraba su ciclo del sueño. Mencionó haber recibido tratamiento con antihistamínicos y pregabalina, sin presentar mejoría clínica.

En la exploración física, se encontraron placas numulares de aspecto eritematoso y violáceo, en fase de descamación, algunas con excoriación y costra serohemática en el centro. Las lesiones se ubicaban principalmente en la espalda, los flancos y los miembros superiores (figura 3).

Se solicitó una biopsia de las lesiones, la cual mostró focos de paraqueratosis, acantosis, con discreta espongiosis e infiltrado linfoide perivascular superficial, con frecuentes eosinófilos y algunos melanófagos (figura 4). En el estudio de inmunohistoquímica, se encontró una población mixta con discreto predominio de $\mathrm{CD}_{3}+$, $\mathrm{CD}_{5+}$, CD2+ y CD7+. Además de la biopsia, se solicitó un hemograma, que evidenció eosinofilia $\left(1020 / \mathrm{mm}^{3}\right)$, sin otras alteraciones en los demás valores.
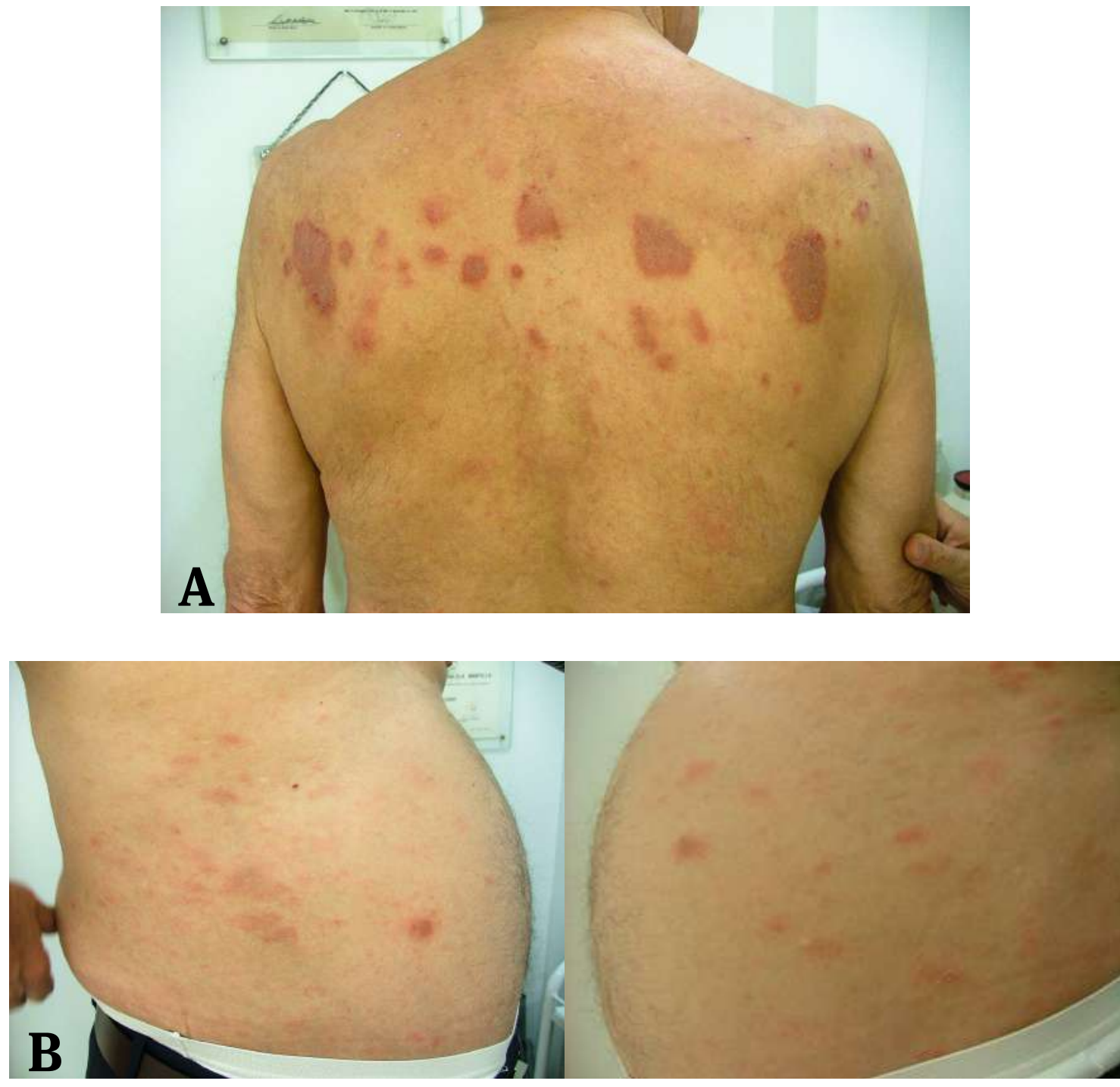

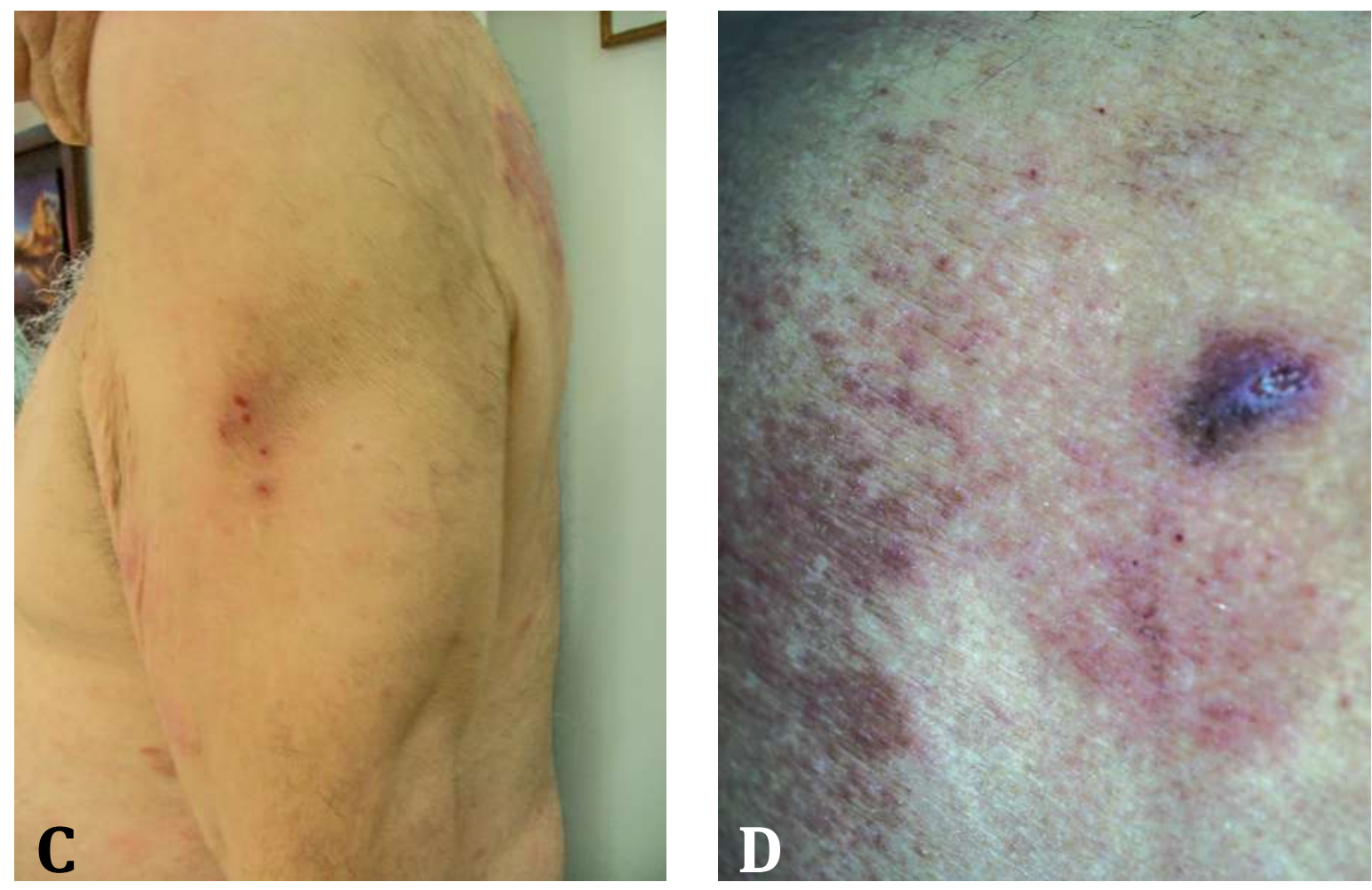

Figura 3. Placas numulares de aspecto eritematoso y violáceo, en fase descamativa, con estigmas de rascado presente, localizadas en la espalda, en ambos flancos y en los miembros superiores. A) Vista dorsal. B) Flancos. C) Brazo izquierdo. D) Brazo derecho.
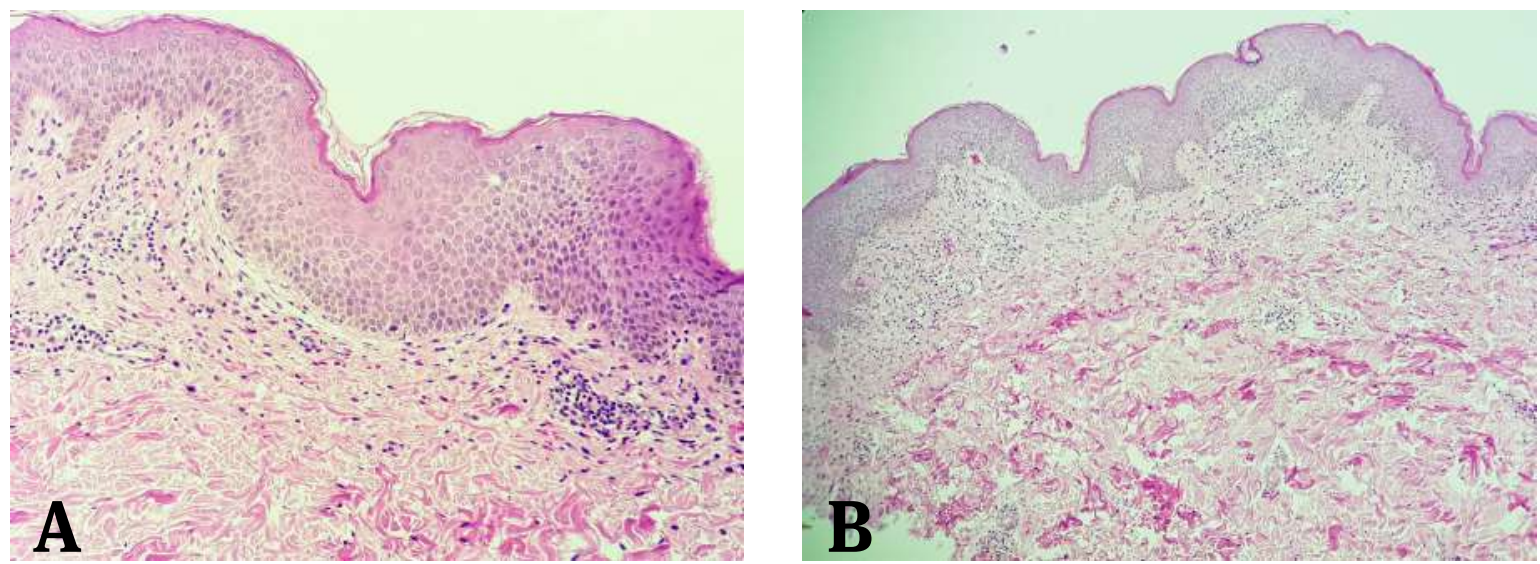

Figura 4. Caso 3. Dermatitis eccematosa crónica. Epidermis con discreta hiperqueratosis, pequeños focos de paraqueratosis, acantosis y ligera espongiosis. En la dermis superior se observa infiltrado inflamatorio, linfoide, perivascular superficial. Tinción con hematoxilina y eosina. A) Fotografía tomada con aumento de 40x. B) Fotografía tomada con aumento de 20x. 
Con base en esto, se formuló el diagnóstico de eccema numular del anciano y se inició tratamiento con talidomida, en una dosis de $100 \mathrm{mg}$ cada noche durante dos semanas y, luego, $50 \mathrm{mg}$ cada noche durante dos semanas más. Además, se inició fototerapia UVA-1 y se sustituyó el jabón regular por un limpiador sin jabón (detergente sintético o syndet) para el baño diario, acompañado de hidrocortisona en crema al $1 \%$ en la noche.

A los 15 días, el paciente consultó por persistencia del prurito y de las lesiones en la piel. En las pruebas analíticas de control, se evidenció elevación de la inmunoglobulina E ( IgE) (2,024 UI/ml), por lo que se continuó con talidomida, en una dosis de $100 \mathrm{mg} /$ día, durante 30 días más, acompañada de sesiones de fototerapia UVA-1 tres veces por semana, hasta completar el ciclo de talidomida, y prednisona oral, en una dosis de 50 $\mathrm{mg} / \mathrm{d}$, durante 7 días; luego, $25 \mathrm{mg} /$ día, durante 7 días; $\mathrm{y}$, posteriormente, $25 \mathrm{mg}$ interdiarios, durante otros 7 días.

A los 30 días, se observó una mejoría significativa de las lesiones en la piel y el paciente refería disminución del prurito, que persistía en los miembros inferiores.
En la exploración física, únicamente se encontró una placa numular, eritematosa, de bordes bien definidos y con costras brillantes en la superficie, sobre el muslo derecho. Se continuó el tratamiento con talidomida, en una dosis de $50 \mathrm{mg} / \mathrm{d}$, durante 30 días, una sesión semanal de fototerapia UVB de banda estrecha (UVB-NB) y aplicación tópica de dipropionato de betametasona sobre las lesiones, dos veces al día.

En los controles posteriores, se observaron únicamente placas residuales hiperpigmentadas y de bordes bien definidos, en la espalda y los flancos. En los exámenes de laboratorio de control se evidenció la normalización de los eosinófilos en la sangre periférica y la persistencia de la elevación de $\operatorname{IgE}$ (350 UI/ml). Ante la persistencia del prurito, se administraron también montelukast y desloratadina por vía oral, fototerapia UVA-1 y dipropionato de betametasona tópico, sin lograrse un adecuado control del síntoma. Finalmente, se administró gabapentina, en una dosis de $400 \mathrm{mg}$ en la noche, con lo cual se logró un mejor control del prurito. El paciente siguió recibiendo este fármaco por tres años y medio, aproximadamente, sin presentar nuevas lesiones en la piel hasta la actualidad (figura 5).
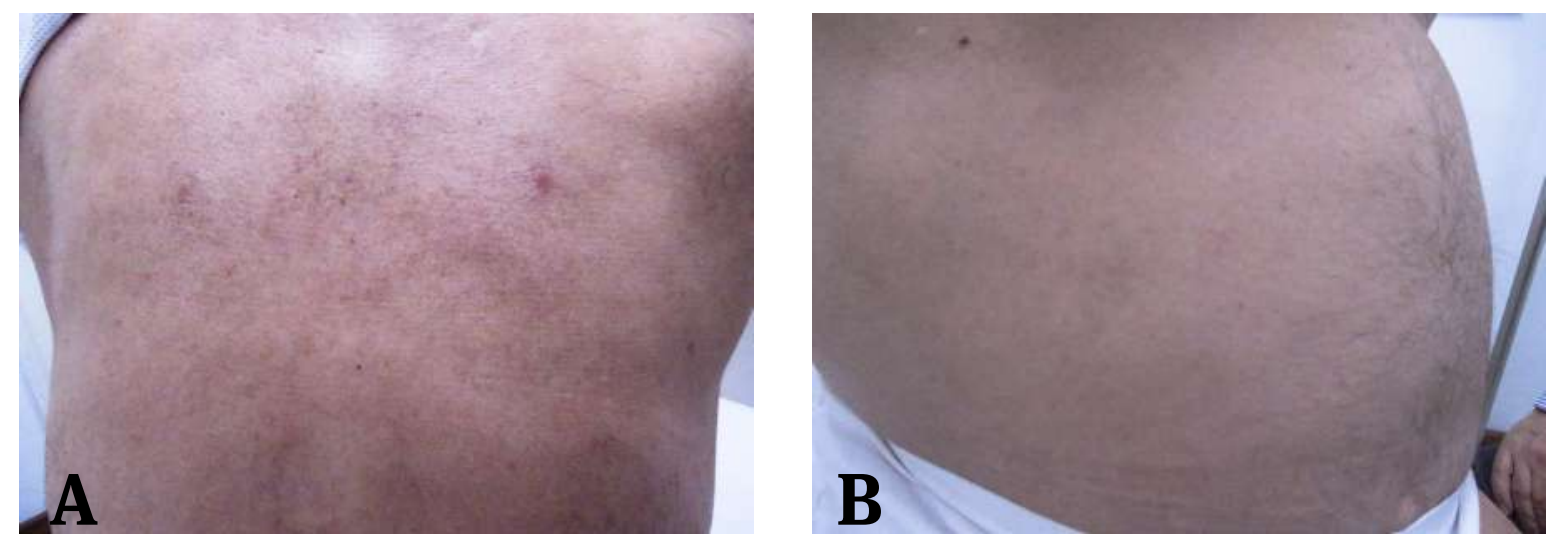

Figura 5. Resolución de las lesiones en piel. A) Vista dorsal. B) Flanco derecho. C) Brazo derecho. 


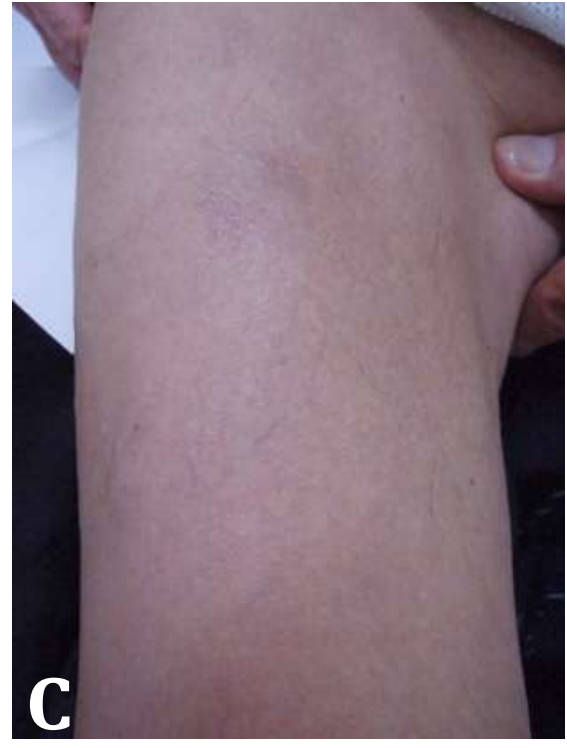

\section{DISCUSIÓN}

El eccema numular, también conocido como eccema discoide, fue descrito clínicamente por primera vez por Rayer, en 1845, y Devergie, en 1857, introdujo el nombre de la entidad como es conocida en la actualidad ${ }^{(1)}$.

Se trata de una enfermedad crónica, recidivante, que se presenta con placas eccematosas, ovaladas o circulares en forma de moneda, pruriginosas, de tamaño variable y bordes papulovesiculares definidos. En la fase aguda se pueden presentar pústulas, supuración y excoriaciones, mientras que en la fase crónica predominan las lesiones liquenificadas y con descamación (2). Las lesiones suelen presentarse predominantemente en las superficies extensoras de los miembros superiores, aunque también es común encontrar lesiones en los miembros inferiores, en el tronco y, con menor frecuencia, en el rostro.

En la histopatología se observan cambios inespecíficos, como edema intercelular en la epidermis, con vesículas espongióticas, asociado a infiltrado linfohistiocitario de predominio perivascular en la dermis superficial (3) (figura 4).

En la mayoría de los estudios se ha demostrado una mayor prevalencia en los varones; sin embargo, en un estudio unicéntrico transversal realizado en Tailandia por Sukhum, et al., hubo predominio de esta entidad en el sexo femenino, en el $67 \%$. Asimismo, Cowan y
Hellgren describieron dos picos de incidencia: entre los 20 y los 60 años y entre los 55 y los 65 años, respectivamente ${ }^{(4)}$.

A pesar de que el eccema numular es una entidad que se conoce hace muchos años, su etiología no ha sido del todo dilucidada. Hasta el momento, se ha postulado que se trata de una enfermedad multifactorial, en la que intervienen factores emocionales, nutricionales, ambientales, infecciosos, farmacológicos y ciertos alérgenos e irritantes. La asociación entre la dermatitis atópica y el eccema numular ha sido controversial; a pesar de que ciertos autores mencionan que no existe una relación entre estas dos enfermedades, algunos de nuestros pacientes presentaron niveles elevados de IgE $(4,5)$. En cuanto a los alérgenos propuestos como causantes y agravantes del eccema numular, se encuentran el sulfato de níquel, el dicromato de potasio, el cloruro de cobalto, la etilendiamina, la neomicina, el oro y el cromo ${ }^{(6)}$.

También se ha propuesto que las infecciones pueden favorecer el desarrollo y la resistencia al tratamiento del eccema numular. Tanaka, et al., presentan el caso de un paciente con diagnóstico de eccema numular tratado con corticoides y antihistamínicos durante dos años sin mejoría, en quien luego se diagnosticó una infección odontogénica grave, por pulpitis y caries en múltiples piezas dentarias. Después del tratamiento odontológico con extracción dental, las lesiones cutáneas se resolvieron y no hubo recurrencia de la enfer$\operatorname{medad}^{(7)}$.

Por otro lado, se ha planteado que los pacientes con eccema discoide, al igual que aquellos con dermatitis atópica, presentan tasas de colonización con Staphylococcus aureus más altas que las de la población general; en ellos existe una correlación positiva entre la gravedad de la enfermedad y la colonización por $S$. aureus ${ }^{(8)}$. Algunos medicamentos, como la metildopa (9) y la asociación de ribavirina e interferón azb o peginterferón $\alpha_{2} b$, se han propuesto como posibles factores desencadenantes de eccema numular ${ }^{(10)}$. Moore, et al., reportaron el caso de un paciente varón de 50 años, con hepatitis $C$ crónica y tratado con ribavirina en cápsulas e interferón $\alpha_{2} b$ por vía subcutánea, que a los cuatro meses de iniciado el tratamiento farmacológico desarrolló lesiones sugestivas de eccema numular en el sitio de la inyección en la región anterior de los muslos y que, posteriormente, se generalizaron a los miembros superiores, el tronco y el rostro ${ }^{(11)}$. La enfermedad puede aparecer durante cualquier temporada del año; sin embargo, existen datos acerca de las exacerbaciones durante el verano y el invierno, por lo que también se le llama "eccema de invierno" (12). 
Hasta el momento, no existe una directriz de manejo para el eccema discoide. En la mayoría de los casos, se reporta la resolución de las lesiones con corticoides tópicos de media y alta potencia, emolientes y antihistamínicos orales para el manejo del prurito. Además, se ha informado el uso tópico de inhibidores de la calcineurina y fototerapia con luz UVB-NB ${ }^{(13)}$.

Para los casos graves, se ha propuesto emplear tratamiento sistémico con diversos agentes. Sin embargo, hasta el momento no contamos con los estudios que validen su efectividad y seguridad, y el uso de la gran mayoría de estos fármacos se deriva de la información obtenida de los estudios sobre dermatitis atópica ${ }^{(14)}$. Entre los medicamentos propuestos, se encuentran la ciclosporina, la azatioprina y el micofenolato de mofetilo. El metotrexato parece ser una opción prometedora; se han reportado casos tanto en adultos como en niños, que demuestran que es eficaz y con pocos efectos secundarios (con mayor frecuencia náuseas, vómito y diarrea) ${ }^{(15)}$.

Existe poca evidencia científica que respalde el uso del metrotexato para el tratamiento del eccema numular. Sin embargo, al hacer la revisión bibliográfica, encontramos un estudio observacional retrospectivo realizado en Madrid, España, donde se dio tratamiento con metotrexato oral a 28 niños con eccema numular grave, desde enero hasta marzo de 2007. En dicho estudio, se evaluó la reacción después de un mes de iniciado el tratamiento y, posteriormente, cada tres meses. Al finalizar el estudio, después de 13,4 meses, el 35,7 \% de los pacientes presentó resolución completa de las lesiones en la piel, el 46,4 \% tuvo una importante mejoría y el 14,3\% presentó una mejoría leve. Se reportó una incidencia muy baja de efectos secundarios con esta modalidad de tratamiento ${ }^{(15)}$.

Además, en otro estudio observacional retrospectivo realizado en Melbourne, Australia, se evaluó el resultado del tratamiento con metrotexato en 25 pacientes pediátricos con eccema numular moderado a intenso. Al finalizar el estudio, el $64 \%$ de los pacientes obtuvo una resolución completa de las lesiones dérmicas y pudo terminar el tratamiento con metrotexato después de un promedio de 10,5 meses de duración. Cabe resaltar que no se reportaron efectos secundarios serios por el uso de este medicamento durante el estudio ${ }^{(14)}$.

\section{CONCLUSIONES}

Los casos aquí presentados pretenden divulgar la información sobre la historia natural del eccema numular y los factores asociados, así como el proceso diagnóstico y el tratamiento de esta enfermedad.

Dada su naturaleza, el eccema numular es una enfermedad poco diagnosticada. Generalmente, los pacientes reciben múltiples tratamientos previos al diagnóstico definitivo, el cual se basa principalmente en la presentación clínica, ya que los resultados de la histopatología son poco específicos. Sin embargo, la presentación clínica es variada. En los pacientes aquí presentados, se observaron lesiones numulares generalizadas (en todo el cuerpo) y con tendencia a ser simétricas, acompañadas de prurito intenso, lo cual disminuye la calidad de vida de los pacientes.

El eccema numular es una enfermedad crónica, en la que son frecuentes las recidivas. El tratamiento se basa principalmente en medicamentos tópicos y, eventualmente, orales. En muchas ocasiones, se requiere más de un medicamento para lograr la resolución de las lesiones.

Finalmente, cabe mencionar que hacen falta estudios que determinen con exactitud el manejo farmacológico y su duración.

\section{REFERENCIAS}

1. Martinez-Blanco J, García-González V, GonzálezGarcía J, Suárez-Castañón C. [Nummular dermatitis: report of two cases in children]. Arch Argent Pediatr. 2016;114(4):e241-4. doi: 10.5546/aap.2016. e241.

2. Silverberg NB. Typical and atypical clinical appearance of atopic dermatitis. Clin Dermatol. 2017;35(4):354-9. doi: 10.1016/j.clindermatol.2017.03.007.

3. Bonamonte D, Foti C, Vestita M, Ranieri LD, Angelini G. Nummular eczema and contact allergy: a retrospective study. Dermatitis. 2012;23(4):153-7. doi: 10.1097/DER.obo13e31826od5ao.

4. Jiamton S, Tangjaturonrusamee C, Kulthanan K. Clinical features and aggravating factors in nummular eczema in Thais. Asian Pacific J Allergy Immunol. 2013;31(1):36-42.

5. Sirot G. Nummular eczema. Semin Dermatol. 1983;2:68-74.

6. Krupa Shankar DS, Shrestha S. Relevance of patch testing in patients with nummular dermatitis. Indian J Dermatol Venereol Leprol. 2005;71(6):406-8.

7. Tanaka T, Satoh T, Yokozeki H. Dental infection associated with nummular eczema as an overlooked focal infection. J Dermatol. 2009;36(8):462-5. doi: 10.1111/j.1346-8138.2009.00677.x. 
8. Kim WJ, Ko HC, Kim MB, Kim DW, Kim JM, Kim BS. Features of Staphylococcus aureus colonization in patients with nummular eczema. Br J Dermatol. 2013;168(3):658-60. doi: 10.1111/j.13652133.2012.11072.x.

9. Church R. Eczema provoked by methyl dopa. Br J Dermatol. 1974;91(4):373-8.

10. Shen Y, Pielop J, Hsu S. Generalized nummular eczema secondary to peginterferon Alfa-2b and ribavirin combination therapy for hepatitis $\mathrm{C}$ infection. Arch Dermatol. 2005;141(1):102-3. doi: 10.1001/archderm.141.1.102-b

11. Moore MM, Elpern DJ, Carter DJ. Severe, generalized nummular eczema secondary to interferon alfa-2b plus ribavirin combination therapy in a patient with chronic hepatitis $\mathrm{C}$ virus infection. Arch Dermatol. 2004;140(2):215-7.

12. Cowan MA. Nummular eczema. A review, follow-up and analysis of a series of 325 cases. Acta Derm Venereol. 1961;41:453-60. doi: 102340/0001555541453460.

13. Coulson I. Discoid eczema. En: Lebwohl MG, Heymann WR, Berth-Jones J, Coulson I (editores). Treatment of Skin Disease: Comprehensive Therapeutic Strategies. Filadelfia: Elsevier Saunders; 2014. p. 190-3.

14. Roberts H, Orchard D. Methotrexate is a safe and effective treatment for paediatric discoid (nummular) eczema: A case series of 25 children. Australas J Dermatol. 2010;51(2):128-30. doi: 10.1111/j.1440-0960.2010.00634.x.

15. Knöpfel N, Noguera-Morel L, Hernández-Martín A, Torrelo A. Methotrexate for severe nummular eczema in children: Efficacy and tolerability in a retrospective study of 28 patients. Pediatr Dermatol. 2018;35(5):611-5. doi: 10.1111/pde.13568. 